\title{
Rainbow matchings in properly-colored hypergraphs
}

\author{
Hao Huang* \\ Department of Math and CS, \\ Emory University, \\ Atlanta, GA, USA. \\ hao. huang@emory.edu
}

\author{
Tong Li \\ Department of Mathematics, \\ Shandong University, \\ Jinan, China. \\ tongli121@163.com
}

\author{
Guanghui Wang ${ }^{\dagger}$ \\ Department of Mathematics, \\ Shandong University, \\ Jinan, China. \\ ghwang@sdu.edu.cn
}

Submitted: Aug 28, 2018; Accepted: Dec 31, 2018; Published: Jan 11, 2019

(C) The authors. Released under the CC BY-ND license (International 4.0).

\begin{abstract}
A hypergraph $H$ is properly colored if for every vertex $v \in V(H)$, all the edges incident to $v$ have distinct colors. In this paper, we show that if $H_{1}, \ldots, H_{s}$ are properly-colored $k$-uniform hypergraphs on $n$ vertices, where $n \geqslant 3 k^{2} s$, and $e\left(H_{i}\right)>\left(\begin{array}{l}n \\ k\end{array}\right)-\left(\begin{array}{c}n-s+1 \\ k\end{array}\right)$, then there exists a rainbow matching of size $s$, containing one edge from each $H_{i}$. This generalizes some previous results on the Erdös Matching Conjecture.
\end{abstract}

Mathematics Subject Classifications: 05C70, 05D05

\section{Introduction}

A $k$-uniform hypergraph is a pair $H=(V, E)$, where $V=V(H)$ is a finite set of vertices, and $E=E(H) \subseteq\left(\begin{array}{l}V \\ k\end{array}\right)$ is a family of $k$-element subsets of $V$ called edges. A matching in a hypergraph $H$ is a collection of vertex-disjoint edges. The size of a matching is the number of edges in the matching. The matching number $\nu(H)$ is the maximum size of a matching in $H$. In 1965, Erdös [4] asked to determine the maximum number of edges that could appear in a $k$-uniform $n$-vertex hypergraph $H$ with matching number $\nu(H)<s$, for given integer $s \leqslant \frac{n}{k}$. He conjectured that the problem has two extremal constructions.

* Research supported in part by the Collaboration Grants from the Simons Foundation.

${ }^{\dagger}$ Research supported by NNSF (No. 11471193, 11631014) 
The first one is a hyper-clique consisting of all the $k$-subsets on $k s-1$ vertices. The other one is a $k$-uniform hypergraph on $n$ vertices containing all the edges intersecting a fixed set of $s-1$ vertices. Erdős posed the following conjecture:

Conjecture 1 ([4]). Every $k$-uniform hypergraph $H$ on $n$ vertices with matching number $\nu(H)<s \leqslant \frac{n}{k}$ satisfies $e(H) \leqslant \max \left\{\left(\begin{array}{c}k s-1 \\ k\end{array}\right),\left(\begin{array}{c}n \\ k\end{array}\right)-\left(\begin{array}{c}n-s+1 \\ k\end{array}\right)\right\}$.

The case $s=1$ is the classic Erdös-Ko-Rado Theorem [6]. The graph case $(k=2)$ was verified in [5] by Erdős and Gallai. The problem seems to be significantly harder for hypergraphs. When $k=3$, Frankl, Rödl and Ruciński [11] proved the conjecture for $s \leqslant \frac{n}{4}$. Euczak and Mieczkowska [14] proved it for sufficiently large $s$. The $k=3$ case was finally settled by Frankl [8]. For general $k$, a short calculation shows that when $s \leqslant \frac{n}{k+1}$, we always have $\left(\begin{array}{l}n \\ k\end{array}\right)-\left(\begin{array}{c}n-s+1 \\ k\end{array}\right)>\left(\begin{array}{c}k s-1 \\ k\end{array}\right)$. For this range, the second construction is believed to be optimal. Erdős [4] proved the conjecture for $n \geqslant n_{0}(k, s)$. Bollobás, Daykin and Erdős [2] proved the conjecture for $n>2 k^{3}(s-1)$. Huang, Loh and Sudakov [12] improved it to $n \geqslant 3 k^{2} s$, which was further improved to $n \geqslant 3 k^{2} s / \log k$ by Frankl, Łuczak and Mieczkowska [10]. On the other hand, in an unpublished note, Füredi and Frankl proved the conjecture for $n \geqslant c k s^{2}$, Frankl [7] improved all the range above to $n \geqslant(2 s-1) k-s+1$. Currently the best range is $n \geqslant \frac{5}{3} s k-\frac{2}{3} s$ by Frankl and Kupavskii $[9]$.

In this paper, we consider a generalization of Erdős Matching Conjecture to properlycolored hypergraphs. A hypergraph $H$ is properly colored if for every vertex $v \in V(H)$, all edges incident to $v$ are colored differently. A rainbow matching in a properly-colored hypergraph $H$ is a collection of vertex disjoint edges with pairwise different colors. The size of a rainbow matching is the number of edges in the matching. The rainbow matching number, denoted by $\nu_{r}(H)$, is the maximum size of a rainbow matching in $H$. Motivated by the Erdős Matching Conjecture, we consider the following problem: how many edges can appear in a properly-colored $k$-uniform hypergraph $H$ such that its rainbow matching number satisfies $\nu_{r}(H)<s \leqslant \frac{n}{k}$ ? In fact, it is called Rainbow Turán problem and is well studied in [13]. Note that here if we let $H$ be rainbow, that is, every edge of $H$ receives distinct colors, then we obtain the original Erdős Matching Conjecture.

More generally, let $H_{1}, \ldots, H_{s}$ be properly-colored $k$-uniform hypergraphs on $n$ vertices, a rainbow matching of size $s$ in $H_{1}, \ldots, H_{s}$ is a collection of vertex disjoint edges $e_{1}, \ldots, e_{s}$ with pairwise different colors, where $e_{1} \in E\left(H_{1}\right), \ldots, e_{s} \in E\left(H_{s}\right)$. For simplicity, we call it an $s$-rainbow matching. Then what is the minimum $M$, such that by assuming $e\left(H_{i}\right)>M$ for every $i$, it guarantees the existance of an $s$-rainbow matching?

In this paper, we prove the following result, which generalizes Theorem 1.2 and Theorem 3.3 of [12].

Theorem 2. Let $H_{1}, \ldots, H_{s}$ be properly-colored $k$-uniform hypergraphs on $n$ vertices. If $n \geqslant 3 k^{2} s$ and every $e\left(H_{i}\right)>\left(\begin{array}{l}n \\ k\end{array}\right)-\left(\begin{array}{c}n-s+1 \\ k\end{array}\right)$, then there exists an s-rainbow matching in $H_{1}, \ldots, H_{s}$. 


\section{Preliminary results}

In this section, we list some preliminary results about "rainbow" hypergraphs, which is a special case of properly-colored hypergraphs. In the next section, we will prove our main theorem with the help of these results. A hypergraph $H$ is rainbow if the colors of any two edges in $E(H)$ are different. From now on, when we say an edge $e$ is disjoint from a collection of edges, it means that not only $e$ is vertex-disjoint from those edges, but it also has a color different from the colors of all these edges. We start by the following lemma for graphs. Note that here although each $G_{i}$ is rainbow, a color may appear in more than one $G_{i}$ 's.

Lemma 3. Let $G_{1}, \ldots, G_{s}$ be rainbow graphs on $n$ vertices. If $n \geqslant 5$ s and $e\left(G_{i}\right)>$ $\left(\begin{array}{l}n \\ 2\end{array}\right)-\left(\begin{array}{c}n-s+1 \\ 2\end{array}\right)$, then there exists an s-rainbow matching in $G_{1}, \ldots, G_{s}$.

Proof. We do induction on $s$. The base case $s=1$ is trivial. For every vertex $v \in V\left(G_{i}\right)$ and $j \neq i$, let $G_{v}^{j}$ be the subgraph of $G_{j}$ induced by the vertex set $V\left(G_{j}\right) \backslash\{v\}$. Since there are at most $n-1$ edges containing $v$ in $E\left(G_{j}\right)$, we have $e\left(G_{v}^{j}\right) \geqslant e\left(G_{j}\right)-(n-1)>$ $\left(\begin{array}{l}n \\ 2\end{array}\right)-\left(\begin{array}{c}n-s+1 \\ 2\end{array}\right)-(n-1)=\left(\begin{array}{c}n-1 \\ 2\end{array}\right)-\left(\begin{array}{c}(n-1)-(s-1)+1 \\ 2\end{array}\right)$. By induction, there exists an $(s-1)-$ rainbow matching $\left\{e_{j}\right\}_{j \neq i}$ in $\left\{G_{v}^{j}\right\}_{j \neq i}$, which spans $2(s-1)$ vertices. So if some $G_{i}$ has a vertex $v$ with degree greater than $3(s-1)$, then there exists an edge $e$ in $G_{i}$ which contains $v$ and disjoint from the edges of the $(s-1)$-rainbow matching, which produces an $s$-rainbow matching. Hence we may assume that the maximum degree of each $G_{i}$ is at most $3(s-1)$.

Now pick an arbitrary edge $u v$ in $G_{1}$. Assume the color of $u v$ is $c(u v)$. Then we delete the vertices $u, v$ and the edge colored by $c(u v)$ in $G_{2}, \ldots, G_{s}$. Denote the resulting graphs by $G_{2}^{\prime}, \ldots, G_{s}^{\prime}$. We can see that when $n \geqslant 5 s$, for each $i \in\{2, \ldots, s\}$, we have $e\left(G_{i}^{\prime}\right)>\left(\begin{array}{c}n \\ 2\end{array}\right)-\left(\begin{array}{c}n-s+1 \\ 2\end{array}\right)-2 \cdot 3(s-1)-1>\left(\begin{array}{c}n-2 \\ 2\end{array}\right)-\left(\begin{array}{c}(n-2)-(s-1)+1 \\ 2\end{array}\right)$. By induction on $s$, there exists an $(s-1)$-rainbow matching in the graphs $G_{2}^{\prime}, \ldots, G_{s}^{\prime}$. Taking these $s-1$ edges with the edge $u v$, we obtain an $s$-rainbow matching in $G_{1}, \ldots, G_{s}$.

Lemma 4. Let $H_{1}, \ldots, H_{s}$ be rainbow $k$-uniform hypergraphs on $n$ vertices. If $n \geqslant 3 k^{2} s$ and $e\left(H_{i}\right)>\left(\begin{array}{l}n \\ k\end{array}\right)-\left(\begin{array}{c}n-s+1 \\ k\end{array}\right)$, then there exists an s-rainbow matching in $H_{1}, \ldots, H_{s}$.

Proof. We do induction on both $k$ and $s$. According to Lemma 3, the case $k=2$ holds for every $s$ and $n \geqslant 5 s$. And for every $k$, the case $s=1$ is trivial. We first consider the situation when some $H_{i}$ has a vertex $v$ with degree greater than $k(s-1)\left(\begin{array}{l}n-2 \\ k-2\end{array}\right)+s-1$. For every vertex $v \in V\left(H_{i}\right)$ and $j \neq i$, let $H_{v}^{j}$ be the subgraph of $H_{j}$ induced by the vertex set $V\left(H_{j}\right) \backslash\{v\}$. Since there are at most $\left(\begin{array}{l}n-1 \\ k-1\end{array}\right)$ edges containing $v$ in $E\left(H_{j}\right)$, we have $e\left(H_{v}^{j}\right) \geqslant e\left(H_{j}\right)-\left(\begin{array}{l}n-1 \\ k-1\end{array}\right)>\left(\begin{array}{l}n \\ k\end{array}\right)-\left(\begin{array}{c}n-s+1 \\ k\end{array}\right)-\left(\begin{array}{l}n-1 \\ k-1\end{array}\right)=\left(\begin{array}{c}n-1 \\ k\end{array}\right)-\left(\begin{array}{c}(n-1)-(s-1)+1 \\ k\end{array}\right)$. By inductive hypothesis for the case $(n-1, k, s-1)$, there exists an $(s-1)$-rainbow matching $\left\{e_{j}\right\}_{j \neq i}$ in $\left\{H_{v}^{j}\right\}_{j \neq i}$, which spans $k(s-1)$ vertices. So if some $H_{i}$ has a vertex $v$ with degree greater than $k(s-1)\left(\begin{array}{l}n-2 \\ k-2\end{array}\right)+s-1$, then there exists an edge $e$ in $E\left(H_{i}\right)$ which contains $v$ and disjoint from the edges of the $(s-1)$-rainbow matching, which produces an $s$-rainbow matching. Hence we may assume that the maximum degree in each hypergraph $H_{i}$ is at most $k(s-1)\left(\begin{array}{l}n-2 \\ k-2\end{array}\right)+s-1$. 
By induction on $s$, we know that for every $i$ there exists an $(s-1)$-rainbow matching in the hypergraphs $\left\{H_{j}\right\}_{j \neq i}$, spanning $k(s-1)$ vertices. If for some $i$, the $s$-th largest degree of $H_{i}$ is at most $2(s-1)\left(\begin{array}{l}n-2 \\ k-2\end{array}\right)+s-1$, then the sum of degrees of these $k(s-1)$ vertices in $H_{i}$ is at most

$$
\begin{aligned}
& (s-1)\left[k(s-1)\left(\begin{array}{l}
n-2 \\
k-2
\end{array}\right)+s-1\right]+(s-1)(k-1)\left[2(s-1)\left(\begin{array}{l}
n-2 \\
k-2
\end{array}\right)+s-1\right] \\
= & (3 k-2)(s-1)^{2}\left(\begin{array}{l}
n-2 \\
k-2
\end{array}\right)+(s-1)^{2} k .
\end{aligned}
$$

Since $n \geqslant 3 k^{2} s$, we have $e\left(H_{i}\right)>\left(\begin{array}{l}n \\ k\end{array}\right)-\left(\begin{array}{c}n-s+1 \\ k\end{array}\right)>(s-1)^{2}\left(3 k-\frac{1}{2}\right)\left(\begin{array}{l}n-2 \\ k-2\end{array}\right)>(3 k-$ $2)(s-1)^{2}\left(\begin{array}{l}n-2 \\ k-2\end{array}\right)+(s-1)^{2} k+s-1$, which guarantees the existence of an edge in $H_{i}$ which is disjoint from the previous $(s-1)$-rainbow matching in $\left\{H_{j}\right\}_{j \neq i}$, which produces an $s$-rainbow matching. So we may assume that each $H_{i}$ contains at least $s$ vertices with degree above $2(s-1)\left(\begin{array}{l}n-2 \\ k-2\end{array}\right)+s-1$.

Now we may greedily select distinct vertices $v_{i} \in V\left(H_{i}\right)$, such that for each $1 \leqslant$ $i \leqslant s$, the degree of $v_{i}$ in $H_{i}$ exceeds $2(s-1)\left(\begin{array}{l}n-2 \\ k-2\end{array}\right)+s-1$. Consider all the subsets of $V\left(H_{i}\right) \backslash\left\{v_{1}, \ldots, v_{s}\right\}$ which together with $v_{i}$ form an edge of $H_{i}$. Denote the $(k-1)$-uniform hypergraph by $H_{i}^{\prime}$. Then $e\left(H_{i}^{\prime}\right)>2(s-1)\left(\begin{array}{l}n-2 \\ k-2\end{array}\right)+s-1-(s-1)\left(\begin{array}{l}n-2 \\ k-2\end{array}\right)>\left(\begin{array}{l}n-s \\ k-1\end{array}\right)-\left(\begin{array}{c}n-2 s+1 \\ k-1\end{array}\right)$. By the inductive hypothesis for the case $(n-s, k-1, s)$, there exists an $s$-rainbow matching $\left\{e_{i}\right\}_{1 \leqslant i \leqslant s}$ in $\left\{H_{i}^{\prime}\right\}_{1 \leqslant i \leqslant s}$. Taking the edges $e_{i} \bigcup\left\{v_{i}\right\}$, we obtain an $s$-rainbow matching in $\left\{H_{i}\right\}_{1 \leqslant i \leqslant s}$.

\section{Main Theorem}

In this section we prove our main result, Theorem 2, using induction and Lemma 4.

Proof. We split our proof into two cases.

Case 1: $\boldsymbol{k}=\mathbf{2}$. Now $H_{1}, \ldots, H_{s}$ are properly-colored graphs. We do induction on $s$. The base case $s=1$ is trivial. For every vertex $v \in V\left(H_{i}\right)$ and $j \neq i$, let $H_{v}^{j}$ be the subgraph of $H_{j}$ induced by the vertex set $V\left(H_{j}\right) \backslash\{v\}$. Since there are at most $n-1$ edges containing $v$ in $E\left(H_{j}\right)$, we have $e\left(H_{v}^{j}\right) \geqslant e\left(H_{j}\right)-(n-1)>\left(\begin{array}{l}n \\ 2\end{array}\right)-\left(\begin{array}{c}n-s+1 \\ 2\end{array}\right)-(n-1)=$ $\left(\begin{array}{c}n-1 \\ 2\end{array}\right)-\left(\begin{array}{c}(n-1)-(s-1)+1 \\ 2\end{array}\right)$. By induction, there exists an $(s-1)$-rainbow matching $\left\{e_{j}\right\}_{j \neq i}$ in $\left\{H_{v}^{j}\right\}_{j \neq i}$, which spans $2(s-1)$ vertices. So if some $H_{i}$ has a vertex $v$ of degree greater than $3(s-1)$, then there exists an edge $e$ in $H_{i}$ which contains $v$ and disjoint from the edges of the $(s-1)$-rainbow matching, which produces an $s$-rainbow matching. Hence we may assume the maximum degree in each $H_{i}$ is at most $3(s-1)$.

For every color $c$ in $H_{i}$ and $j \neq i$, let $H_{c}^{j}$ be the subgraph of $H_{j}$ obtained by deleting all the edges colored by $c$ in $E\left(H_{j}\right)$. Since each $H_{j}$ is properly colored, there are at most $\frac{n}{2}$ edges colored by $c$ in $E\left(H_{j}\right)$. So $e\left(H_{c}^{j}\right) \geqslant e\left(H_{j}\right)-\frac{n}{2}>\left(\begin{array}{l}n \\ 2\end{array}\right)-\left(\begin{array}{c}n-s+1 \\ 2\end{array}\right)-\frac{n}{2}>\left(\begin{array}{c}n \\ 2\end{array}\right)-\left(\begin{array}{c}n-(s-1)+1 \\ 2\end{array}\right)$. By induction, there exists an $(s-1)$-rainbow matching $\left\{e_{j}\right\}_{j \neq i}$ in $\left\{H_{v}^{j}\right\}_{j \neq i}$, which spans $2(s-1)$ vertices $u_{1}, \ldots, u_{2(s-1)}$. Also since $H_{i}$ is properly colored, it has at most one edge containing each $u_{j}$ and colored by $c$. So if the number of edges in $H_{i}$ colored by $c$ is greater than 2(s-1), then there exists an edge $e$ in $H_{i}$ colored by $c$ and disjoint from $\left\{e_{j}\right\}_{j \neq i}$, which produces an $s$-rainbow matching. So we can now assume that the number of edges in every color in each $H_{i}$ is at most $2(s-1)$. 
Now pick an arbitrary edge $u v$ in $H_{1}$. Assume the color of $u v$ is $c(u v)$. Then we delete the vertices $u, v$ and all the edges colored by $c(u v)$ in $H_{2}, \ldots, H_{s}$. Denote the resulting graphs by $H_{2}^{\prime}, \ldots, H_{s}^{\prime}$. We can see that when $n \geqslant 7 s$, for each $i \in\{2, \ldots, s\}$, we have $e\left(H_{i}^{\prime}\right)>\left(\begin{array}{c}n \\ 2\end{array}\right)-\left(\begin{array}{c}n-s+1 \\ 2\end{array}\right)-2 \cdot 3(s-1)-2(s-1)>\left(\begin{array}{c}n-2 \\ 2\end{array}\right)-\left(\begin{array}{c}(n-2)-(s-1)+1 \\ 2\end{array}\right)$. By induction on $s$, there exists an $(s-1)$-rainbow matching in the graphs $H_{2}^{\prime}, \ldots, H_{s}^{\prime}$. Taking these $s-1$ edges with the edge $u v$, we obtain an $s$-rainbow matching in $H_{1}, \ldots, H_{s}$.

Case 2: $\boldsymbol{k} \geqslant 3$. We do induction on $s$. The case $s=1$ is trivial. We first consider the situation when some $H_{i}$ has a vertex of degree greater than $k(s-1)\left(\begin{array}{l}n-2 \\ k-2\end{array}\right)+s-1$. For every vertex $v \in H_{i}$ and $j \neq i$, let $H_{v}^{j}$ be the subgraph of $H_{j}$ induced by the vertex set $V\left(H_{j}\right) \backslash\{v\}$. Since there are at most $\left(\begin{array}{l}n-1 \\ k-1\end{array}\right)$ edges containing $v$ in $E\left(H_{j}\right)$, we have $e\left(H_{v}^{j}\right) \geqslant e\left(H_{j}\right)-\left(\begin{array}{l}n-1 \\ k-1\end{array}\right)>\left(\begin{array}{l}n \\ k\end{array}\right)-\left(\begin{array}{c}n-s+1 \\ k\end{array}\right)-\left(\begin{array}{l}n-1 \\ k-1\end{array}\right)=\left(\begin{array}{c}n-1 \\ k\end{array}\right)-\left(\begin{array}{c}(n-1)-(s-1)+1 \\ k\end{array}\right)$. By induction, there exists an $(s-1)$-rainbow matching $\left\{e_{j}\right\}_{j \neq i}$ in $\left\{H_{v}^{j}\right\}_{j \neq i}$, which spans $k(s-1)$ vertices. So if some $H_{i}$ has a vertex $v$ with degree greater than $k(s-1)\left(\begin{array}{l}n-2 \\ k-2\end{array}\right)+s-1$, then there exists an edge $e$ in $E\left(H_{i}\right)$ which contains $v$ and disjoint from the edges of the $(s-1)$-rainbow matching, which produces an $s$-rainbow matching. Hence we may assume the maximum degree in each hypergraph $H_{i}$ is at most $k(s-1)\left(\begin{array}{l}n-2 \\ k-2\end{array}\right)+s-1$.

By induction on $s$, we know that for every $i$ there exists an $(s-1)$-rainbow matching in the hypergraphs $\left\{H_{j}\right\}_{j \neq i}$, spanning $k(s-1)$ vertices. If for some $i$, the $s$-th largest degree of $H_{i}$ is at most $2(s-1)\left(\begin{array}{l}n-2 \\ k-2\end{array}\right)+s-1$, then the sum of degrees of these $k(s-1)$ vertices in $H_{i}$ is at most

$$
(s-1)\left[k(s-1)\left(\begin{array}{l}
n-2 \\
k-2
\end{array}\right)+s-1\right]+(s-1)(k-1)\left[2(s-1)\left(\begin{array}{l}
n-2 \\
k-2
\end{array}\right)+s-1\right]=(3 k-2)(s-
$$
$1)^{2}\left(\begin{array}{c}n-2 \\ k-2\end{array}\right)+(s-1)^{2} k$.

On the other hand, the maximum degree of the subgraph of $H_{i}$ by deleting these $k(s-1)$ vertices is at most $s-1$, otherwise, we can find an $s$-rainbow matching. Since $n \geqslant 3 k^{2} s$, we have $e\left(H_{i}\right)>\left(\begin{array}{l}n \\ k\end{array}\right)-\left(\begin{array}{c}n-s+1 \\ k\end{array}\right)>(s-1)^{2}\left(3 k-\frac{1}{2}\right)\left(\begin{array}{l}n-2 \\ k-2\end{array}\right)>(3 k-2)(s-1)^{2}\left(\begin{array}{l}n-2 \\ k-2\end{array}\right)+(s-1)^{2} k+$ $\frac{(s-1)[n-k(s-1)]}{k}$, which guarantees the existence of an edge in $H_{i}$ disjoint from the previous $(s-1)$-rainbow matching in $\left\{H_{j}\right\}_{j \neq i}$, which produces an $s$-rainbow matching. So we may assume that each $H_{i}$ contains at least $s$ vertices with degree above $2(s-1)\left(\begin{array}{l}n-2 \\ k-2\end{array}\right)+s-1$.

Now we may greedily select distinct vertices $v_{i} \in V\left(H_{i}\right)$, such that for each $1 \leqslant$ $i \leqslant s$, the degree of $v_{i}$ in $H_{i}$ exceeds $2(s-1)\left(\begin{array}{l}n-2 \\ k-2\end{array}\right)+s-1$. Consider all the subsets of $V\left(H_{i}\right) \backslash\left\{v_{1}, \ldots, v_{s}\right\}$ which together with $v_{i}$ form an edge of $H_{i}$. Denote the $(k-1)$-uniform hypergraph by $H_{i}^{\prime}$. Since each $H_{i}$ is properly colored, we can see that each $H_{i}^{\prime}$ is rainbow and $e\left(H_{i}^{\prime}\right)>2(s-1)\left(\begin{array}{l}n-2 \\ k-2\end{array}\right)+s-1-(s-1)\left(\begin{array}{l}n-2 \\ k-2\end{array}\right)>\left(\begin{array}{c}n-s \\ k-1\end{array}\right)-\left(\begin{array}{c}n-2 s+1 \\ k-1\end{array}\right)$. By Lemma 4, there exists an $s$-rainbow matching $\left\{e_{i}\right\}_{1 \leqslant i \leqslant s}$ in $\left\{H_{i}^{\prime}\right\}_{1 \leqslant i \leqslant s}$. Taking the edges $e_{i} \bigcup\left\{v_{i}\right\}$, we obtain an $s$-rainbow matching in $\left\{H_{i}\right\}_{1 \leqslant i \leqslant s}$.

\section{Concluding Remarks}

In this short note, we propose a generalization of the Erdös hypergraph matching conjecture to finding rainbow matchings in properly-colored hypergraphs, and prove Theorem 2 for $s<n /\left(3 k^{2}\right)$. The following conjecture seems plausible. 
Conjecture 5. There exists constant $C>0$ such that if $H_{1}, \ldots, H_{s}$ are properly-colored $k$-uniform hypergraphs on $n$ vertices, with $n \geqslant C k s$ and every $e\left(H_{i}\right)>\left(\begin{array}{l}n \\ k\end{array}\right)-\left(\begin{array}{c}n-s+1 \\ k\end{array}\right)$, then there exists an $s$-rainbow matching in $H_{1}, \ldots, H_{s}$.

Recall that for the special case when each $H_{i}$ is identical and rainbow, Frankl and Kupavskii [9] were able to verify it for $C=5 / 3$. However the proof relies on the technique of shifting, while the property of a hypergraph being properly colored may not be preserved under shifting.

It is tempting to believe that Erdős Matching Conjecture can be extended to properlycolored hypergraphs for the entire range of $s$, that is, once the number of edges in each hypergraph exceeds the maximum of $\left(\begin{array}{l}n \\ k\end{array}\right)-\left(\begin{array}{c}n-s+1 \\ k\end{array}\right)$ and $\left(\begin{array}{c}k s-1 \\ k\end{array}\right)$, then one can find an $s$ rainbow matching. However this is false in general, a simple construction is by taking $s=2$ and $n=2 k$. The maximum of these two expressions is $\left(\begin{array}{c}2 k-1 \\ k\end{array}\right)$, while one can let $H_{1}$ be a rainbow $K_{2 k}^{k}$ with an edge coloring $c_{1}$, and $H_{2}$ be on the same vertex set with edge coloring $c_{2}$, such that $c_{2}(e)=c_{1}([2 k] \backslash e)$. Then clearly each $H_{i}$ contains $\left(\begin{array}{c}2 k \\ k\end{array}\right)>\left(\begin{array}{c}2 k-1 \\ k\end{array}\right)$ edges and there is no 2-rainbow matching. It would be interesting to find constructions for $s$ close to $n / k$, and formulate a complete conjecture for properly-colored hypergraphs.

\section{References}

[1] J. Akiyama, P. Frankl, On the size of graphs with complete-factors, J. Graph Theory, 9(1)(2010), 197-201.

[2] B. Bollobás, D.E. Daykin, P. Erdős, Sets of independent edges of a hypergraph, $Q$. J. Math. Oxf. Ser., (2) 27 (105)(1976), 25-32.

[3] M. Deza, P. Frankl, Erdős-Ko-Rado theorem - 22 years later, SIAM J. Algebr. Discrete Methods, 4(4) (1983), 419-431.

[4] P. Erdős, A problem on independent $r$-tuples, Ann. Univ. Sci. Budapest. Eötvös Sect. Math., 8(1965), 93-95.

[5] P. Erdös, T. Gallai, On the maximal paths and circuits of graphs, Acta Math. Acad. Sci. Hung., 10(1959), 337-357.

[6] P. Erdős, C. Ko, R. Rado, Intersection theorems for systems of finite sets, Quart. J. Math. Oxford Ser., (2)12 (1961), 313-320.

[7] P. Frankl, Improved bounds for Erdős' matching conjecture, J. Combin. Theory Ser. A, 120 (2013), 1068-1072.

[8] P. Frankl, On the maximum number of edges in a hypergraph with a given matching number, Discrete Appl. Math., 216 (2017), 562-581.

[9] P. Frankl, A. Kupavskii, The Erdős Matching Conjecture and concentration inequalities, available at arXiv:1806.08855.

[10] P. Frankl, T. Łuczak, K. Mieczkowska, On matchings in hypergraphs, Electron. J. Combin., 19(2) (2012), \#P42. 
[11] P. Frankl, V. Rödl, A. Ruciński, On the maximum number of edges in a triple system not containing a disjoint family of a given size, Combin. Probab. Comput., 21(2012), 141-148.

[12] H. Huang, P. Loh, B. Sudakov, The size of a hypergraph and its matching number, Combin. Probab. Comput., 21 (2012), 442-450.

[13] P. Keevash, D. Mubayi, B. Sudakov and J. Verstraëte, Rainbow Turán problems, Combin. Probab. Comput., 16 (2007), 109-126.

[14] T. Łuczak, K. Mieczkowska, On Erdős extremal problem on matchings in hypergraphs, J. Combin. Theory Ser. A, 124 (2014), 178-194. 